\title{
THEORETICAL STUDIES OF THE REACTIONS AND SPECTROSCOPY OF RADICAL SPECIES RELEVANT TO COMBUSTION REACTIONS AND DIAGNOSTICS
}

\author{
DOE/ER/14189-3
}

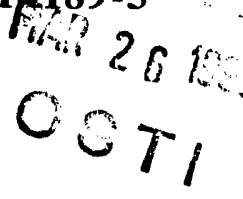

PROGRESS REPORT

DOE/ER/14189--3

PERIOD: 16 MARCH 1992 - 15 MARCH 1993

DE93 010247

\author{
DAVID R. YARKONY
}

DEPARTMENT OF CHEMISTRY

THE JOHNS HOPKINS UNIVERSITY

BALTIMORE, MD 21218

MARCH 1993

PREPARED FOR

THE U. S. DEPARTMENT OF ENERGY

AGREEMENT NO. DE-FG02-91ER14189

\begin{abstract}
NOTICE
This report was prepared as an account of work sponsored by the United States Government. Neither the United States nor the Department of Energy, nor any of their employees, nor any of their contractors, subcontractors, or their employees, makes any warranty, express or implied, or assumes any legal liability or responsibility for the accuracy, completeness, or usefulness of any information, apparatus, product or process disclosed or represents that its use would not infringe privately owned rights.
\end{abstract}

\section{MASTER}

Distribution of this nocumemr is unta da 


\section{ABSTRACT}

This report describes work performed as part of Department of Energy grant DE-FG0291ER14189 during the period 16 March 1992-15 March 1993 and outlines directions for future research. During this period we have developed a new method for determining points on the surface of intersection of two potential energy surfaces corresponding to states of distinct spinmultiplicity. This approach allows any number of geometrical parameters to be fixed while the remainder are optimized to minimize the energy of the point on the crossing surface. This algorithm will significantly enhance our ability to characterize spin-forbidden electronically nonadiabatic processes. Preliminary applications of this algorithm to treat the reaction $\mathrm{CH}\left(\mathrm{X}^{2} \Pi\right)+$ $\mathrm{N}_{2}\left(\mathrm{X}^{1} \Sigma_{\mathrm{g}}^{+}\right) \rightarrow \mathrm{HCN}\left(\mathrm{X}^{1} \Sigma^{+}\right)+\mathrm{N}\left({ }^{4} S\right)$ have been reported. In addition two computational studies are nearing completion:(i) a determination the surfaces of intersection and spin-orbit couplings relevant to the predissociation reaction $\mathrm{N}_{2} \mathrm{O}\left(\mathrm{X}^{1} \Sigma^{+}\right) \rightarrow \mathrm{N}_{2}\left(\mathrm{X}^{1} \Sigma_{\mathrm{g}}^{+}\right)+\mathrm{O}\left({ }^{3} \mathrm{P}\right)$ and the atmospheric quenching reaction $\mathrm{N}_{2}\left(\mathrm{X}^{1} \Sigma_{\mathrm{g}}^{+}\right)+\mathrm{O}\left({ }^{1} \mathrm{D}\right) \rightarrow \mathrm{N}_{2}\left(\mathrm{X}^{1} \Sigma_{\mathrm{g}}^{+}\right)+\mathrm{O}\left({ }^{3} \mathrm{P}_{\mathrm{J}}\right)$ and (ii) a study of the fine-structure distribution in $\mathrm{O}\left({ }^{3} \mathrm{PJ}_{\mathrm{J}}\right)$ produced in the spin-forbidden photodissociation process $\mathrm{OH}\left(\mathrm{X}^{2} \Pi\right)+\mathrm{hv} \rightarrow \mathrm{OH}\left(\mathrm{A}^{2} \Sigma^{+}\right)$ $\rightarrow \mathrm{O}\left({ }^{3} \mathrm{PJ}_{\mathrm{J}}+\mathrm{H}\left({ }^{2} \mathrm{~S}\right)\right.$. This later combined electronic structure/quantum dynamics study is unique in that all the relevant intersurface - nonadiabatic - interactions are determined using MCSCF/CI wavefunctions. 


\section{TECHNICAL, REPORT}

Our research program focusses on studies of spin-forbidden and electronically nonadiabatic processes involving radical species that are relevant to combustion reactions and combustion diagnostics. To study the electronic structure aspects of these processes a unique and powerful system of electronic structure programs, developed over the past nine years, the BROOKLYN codes, is employed. These programs enable us to address questions basic to the understanding of elementary combustion processes not tractable using more standard quantum chemistry codes. Particularly relevant to this research program are the capabilities to

(i) treat the spin-orbit interaction within the context of the full microscopic Breit-Pauli approximation,

(ii) determine the interstate derivative couplings $f_{\alpha}^{\prime J}(\mathbf{R})=\left\langle\Psi_{l}^{0}(\mathbf{r} ; \mathbf{R}) \mid \frac{\partial}{\partial R_{\alpha}} \Psi_{J}^{0}(\mathbf{r} ; \mathbf{R})\right\rangle_{\mathbf{r}}$ that result in the breakdown of the single surface Born Oppenheimer approximation,

(iii) locate surfaces of (actual/avoided) intersection of potential energy surfaces of the same symmetry, and

(iv) locate the minimum energy point on the surface of intersection of two potential energy surfaces of different spin-multiplicity and locate additional points on this crossing surface for which an arbitrary number of internal coordinates are held fixed and the remaining coordinates are optimized to reduce the energy of the crossing point.

\section{Electronic Structure Algorithms}

During the current performance period we have developed

An Algorithm for the Systematic Determination of Points on the Surface of Intersection of Two Potential Energy Surfaces of Distinct Spin-Multiplicity

Two nonrelativistic Born-Oppenheimer potential energy surfaces of distinct space-spin symmetry intersect on a surface of dimension $\mathrm{N}^{\mathrm{I}}-1$ where $\mathrm{N}^{\mathrm{I}}$ is the number of internal nuclear degrees of freedom. Characterization of this entire surface can be quite costly. In many circumstances it suffices to determine only the minimum energy point on the surface of intersection in question. Such a situation is illustrated in the figure below. 


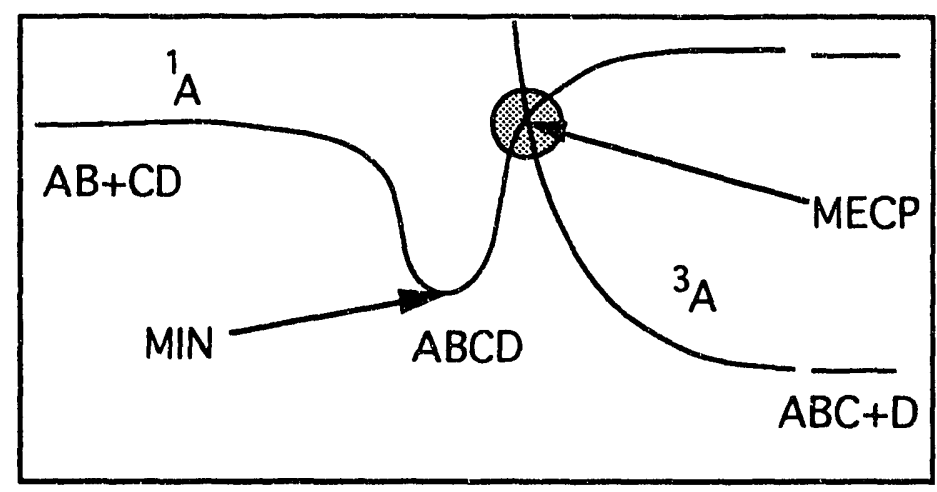

In this case the minimum energy crossing point (MECP) represents the barrier to the spinforbidden process. However favorable situations such as this are not uniformly the case. The figure below depicts a spin-forbidden bimolecular reaction in which much more of the crossing surface is energeticaily accessible.

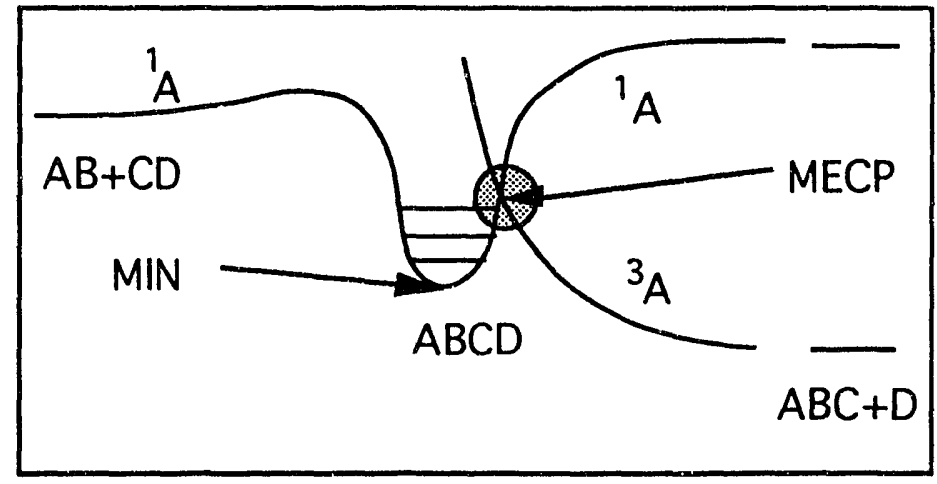

Motivated by this situation an algorithm, employing multiconfiguration self-consistentfield(MCSCF) / configuration interaction(CI) wavefunctions and analytic gradient techniques, has been developed which avoids the determination of the full $\mathrm{N}^{\mathrm{I}}-1$ dimensional surface, while directly locating portions of the crossing surface that are energetically important. The algorithm represents an extension of our previously introduced method ${ }^{1.2}$ for determining the minimum energy point on the surface of intersection of two states of distinct spin-multiplicity. The algorithm is based on the minimization of the Lagrangian function $L^{U}\left(R, \lambda_{0}, \lambda\right)=E_{I}(R)+\lambda_{0}\left[E_{I}(R)-E_{J}(R)\right]$ $+\sum_{k=1}^{M} \lambda_{k} C_{k}(\mathbf{R})$ where $C_{k}(\mathbf{R})$ is any geometrical equality constraint such as $R_{K L}{ }^{2}-a_{K L}{ }^{2}=0$, or $\mathrm{R}_{\mathrm{KL}}^{k=1}{ }^{2}-\mathrm{R}_{\mathrm{MN}}{ }^{2}=0$, with $\mathrm{R}_{\mathrm{KL}}=\mid \mathbf{R}_{\mathrm{K}}-\mathbf{R}_{\mathrm{L}}$ !, and the $\lambda_{0}, \lambda$ are Lagrange multipliers. ${ }^{3}$.

The key aspects of the algorithm are: (i) it is direct, in the sense that the desired constrained minima on the surface of intersection are determined without prior determination of the 
individual potential energy surfaces themselves and (ii) the requisite energy gradients are eavluated using analytic gradient techniques. The details of the formalism can be found in Ref. 3 .

The situation illustrated in the later figure above is expected to be encountered in the spinforbidden reaction $\mathrm{CH}\left(X^{2} \Pi\right)+\mathrm{N}_{2}\left(X^{1} \Sigma_{g}^{\dagger}\right) \rightarrow H C N\left(X^{1} \Sigma^{+}\right)+N\left({ }^{4} S\right)$. Consequently this system was used to demonstrate the efficacy of this new algorithm using a simple $\mathrm{MCSCF} /$ first order $\mathrm{CI}$ description of that reaction. Sections of the crossing surface and the interstate spin-orbit couplings for the ${ }^{4} \mathrm{~A}^{\prime \prime}$ and ${ }^{2} \mathrm{~A}$ " potential energy surfaces were determined in the vicinity of a minimum energy crossing point. $^{3}$

\section{Applications: Spin-forbidden Processes}

(a) Spin-forbidden processes involving $\mathrm{N}_{2} \mathrm{O}$

Motivated by conversations with Dr. Bruce Klemm at the Brookhaven National Laboratory concerning the spin-forbidden predissociation

$$
\mathrm{N}_{2} \mathrm{O}\left(\mathrm{X}^{1} \Sigma^{+}\right) \rightarrow \mathrm{N}_{2}\left(\mathrm{X}^{1} \Sigma_{\mathrm{g}}^{\dagger}\right)+\mathrm{O}\left({ }^{3} \mathrm{P}\right)
$$

and our longstanding interest in the atmospheric quenching reaction

$$
\mathrm{N}_{2}\left(X^{1} \Sigma_{g}^{+}\right)+\mathrm{O}\left({ }^{1} \mathrm{D}\right) \rightarrow \mathrm{N}_{2}\left(\mathrm{X}^{1} \Sigma_{\mathrm{g}}^{+}\right)+\mathrm{O}\left({ }^{3} \mathrm{P}\right)
$$

we have largely completed a study of the crossing surfaces, denoted $\left(1^{1} \mathrm{~A}^{\prime}, 1^{3} \mathrm{~A}^{\prime}\right),\left(1^{1} \mathrm{~A}^{\prime}, 1^{3} \mathrm{~A}^{\prime \prime}\right)$, and $\left(1^{1} \mathrm{~A}^{\prime}, 2^{3} \mathrm{~A}^{\prime \prime}\right)$, corresponding to the intersection the lowest singlet surface of $\mathrm{N}_{2} \mathrm{O}\left(1^{1} \mathrm{~A}^{\prime}\right)$ with the three triplet surfaces correlating with $\mathrm{N}_{2}+\mathrm{O}\left({ }^{3} \mathrm{P}\right)\left(1^{3} \mathrm{~A}^{\prime}, 1,2^{3} \mathrm{~A}^{\prime \prime}\right)$ using multireference configuration interaction wavefunctions comprised of $400,000-600,000$ terms. These crossing surfaces were characterized in the vicinity of their minimum energy crossing points. The minimum energy crossing structures are all linear and thus correspond to $C_{o o v}\left(X^{1} \Sigma^{+}, 3 \Pi\right)$ and $\left(X^{1} \Sigma^{+}, 3 \Sigma^{-}\right)$ intersections. The minimum energy point on the $\left(X^{1} \Sigma^{+},{ }^{3} \Pi\right)$ crossing surface, was found to be $57 \mathrm{kcal} / \mathrm{mol}$ above the $\mathrm{N}_{2} \mathrm{O}\left(\mathrm{X}^{1} \Sigma^{+}\right)$minimum. The minimum energy point on the $\left(\mathrm{X}^{1} \Sigma^{+},{ }^{3} \Sigma^{--}\right)$ crossing surface was found to be $68 \mathrm{kcal} / \mathrm{mol}$ above the $\mathrm{N}_{2} \mathrm{O}\left(\mathrm{X}^{1} \Sigma^{+}\right)$minimum. The $\mathrm{N}-\mathrm{N}$ bond distance is similar at the $\left(X^{1} \Sigma^{+}, 3 \Pi\right)$ and $\left(X^{1} \Sigma^{+}, 3 \Sigma^{-}\right)$minimum energy crossing structures, being $1.116 \AA$ and $1.113 \AA$ respectively, and approximately equal to that in isolated $\mathrm{N}_{2}\left(\mathrm{X}^{1} \Sigma_{\mathrm{g}}^{+}\right)$. The $\mathrm{N}-\mathrm{O}$ bond is $1.72 \AA$ and $1.96 \AA$ at the $\left(X^{1} \Sigma^{+}, 3 \Pi\right)$ and $\left(X^{1} \Sigma^{+}, 3 \Sigma^{-}\right)$minimum energy crossing points 
respectively and is significantly stretched, by over $0.5 \AA$, when compared with its value, $1.184 \AA$, at the equilibrium geometry of $\mathrm{N}_{2} \mathrm{O}\left(\mathrm{X}^{1} \Sigma^{+}\right)$.

The spin-orbit couplings were also evaluated on each of the $\left(1^{1} A^{\prime}, 1^{3} A^{\prime}\right),\left(1^{1} A^{\prime}, 1^{3} A^{\prime \prime}\right)$, and $\left(1^{1} A^{\prime}, 2^{3} A^{\prime \prime}\right)$ crossing surfaces. For collinear geometries the $\left(X^{1} \Sigma^{+}, 3 \Pi\right)$ and $\left(X^{1} \Sigma^{+}, 3 \Sigma^{-}\right)$spinorbit induced couplings are found to be significantly different. The $\left(X^{1} \Sigma^{+},{ }^{3} \Pi\right)$ spin-orbit interaction was found to be $\sim 90 \mathrm{~cm}^{-1}$ at the minimum energy crossing point for the $\left(\mathrm{X}^{1} \Sigma^{+}, 3 \Pi\right)$ intersection while the $\left(\mathrm{X}^{1} \Sigma^{+},{ }^{3} \Sigma^{-}\right)$spin-orbit interaction is only $\sim 8 \mathrm{~cm}^{-1}$ at the minimum energy crossing point for the $\left(\mathrm{X}^{1 \Sigma^{+}}, 3 \Sigma^{-}\right)$intersection.

(b) State Specific Photodissociation of $\mathrm{OH}\left(\mathrm{X}^{2} \Pi\right)$ via $\mathrm{OH}\left(\mathrm{A}^{2} \Sigma^{+}\right)$

In situ detection of nascent $\mathrm{OH}$ is an important problem in studies of combustion processes. Recently several groups, 4, 5,6 including Gray and Farrow at the Sandia Combustion Research Facility ${ }^{5}$ and Crosley and co-workers are SRI International, ${ }^{6}$ have considered using the strongly predissociated $\mathrm{OH}\left(\mathrm{A}^{2} \Sigma^{+}, \mathrm{v}=3\right)$ state in a laser fluorescence detection scheme. In a complementary theoretical work we considered the predissociation of the $\mathrm{OH}\left(\mathrm{A}^{2} \Sigma^{+}, \mathrm{v}=3\right){ }^{7}$ During the current performance period we have extended our studies to consider the fine structure distribution of $\mathrm{O}\left({ }^{3} \mathrm{P}_{\mathrm{J}}\right)$ produced in the photodissociation $\mathrm{OH}\left(\mathrm{X}^{2} \Pi, v=0\right)+\mathrm{hv} \rightarrow \mathrm{OH}\left(\mathrm{A}^{2} \Sigma^{+}, v\right) \rightarrow$ $\mathrm{O}\left({ }^{3} \mathrm{P}_{\mathrm{J}}\right)+\mathrm{H}\left({ }^{2} \mathrm{~S}\right)$ (see the following figure). 


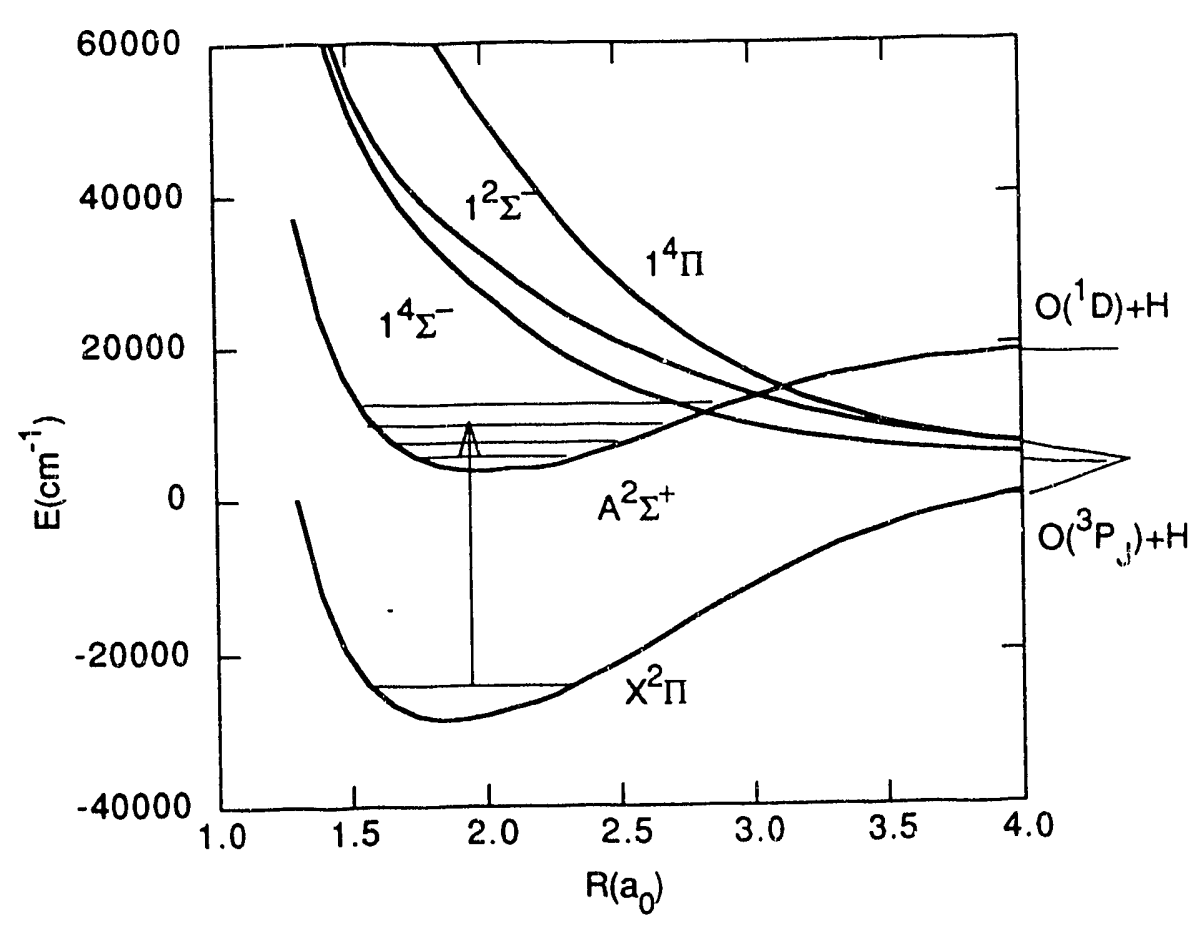

To study this process in a realistic manner it was necessary to determine all the intersurface nonadiabatic - interactions ( 11 unique spin-orbit interactions and 3 coriolus couplings) among the five electronic states in question, $\mathrm{X}^{2} \Pi, 1^{4} \Pi, 1^{4} \Sigma^{-}, 1^{2} \Sigma^{-}$, and $\mathrm{A}^{2} \Sigma^{+}$. This was accomplished using the same large scale configuration interaction wavefunctions employed in our previous treatment of $\mathrm{OH}\left(\mathrm{A}^{2} \Sigma^{+}, \mathrm{v}=3\right)$ predissociation ${ }^{7}$ and represents the first study in which all the relevant nonadiabatic interactions have been determined with such accurate electronic wavefunctions. The photodissociation process was treated using a fully quantum mechanical scattering procedure. Although state specific photodissociation of $\mathrm{OH}$ has been studied in the past ${ }^{8}$ our results permit, for the first time, definite conclusions concerning the influence of the geometry dependence of the nonadiabatic interactions on the $\mathrm{O}\left({ }^{3} \mathrm{PJ}\right)$ branching ratios. It was found that the $\mathrm{O}\left({ }^{3} \mathrm{PJ}\right)$ distributions resulting from photodissociation involving $\mathrm{OH}\left(\mathrm{A}^{2} \Sigma^{+}, \mathrm{v}=4,5\right)$ exhibit the most significant quantum interference effects. $\mathrm{O}\left({ }^{3} \mathrm{P}_{\mathrm{J}}\right)$ distributions for the remaining vibrational levels examined could be determined semiquantiatively using branching ratios obtained from the Fermi Golden Rule. The Golden Rule values for the total decay rates were found to be in good 
agreement with the exact quantum values even for levels with lifetimes on the order of picoseconds.

\section{FUTURE PLANS}

During the forthcoming year we plan to pursue some needed extensions of the research discussed above.

The potential energy surfaces for the reaction $\mathrm{CH}\left(X^{2} \Pi\right)+\mathrm{N}_{2} \rightarrow \mathrm{HCN}\left(X^{1} \Sigma^{+}\right)+\mathrm{N}\left({ }^{4} S\right)$ reaction are currently the object of study in several research groups. We intend to complement these studies with a characterization of the doublet-quartet crossing surface based on the algorithm discussed above and determine the spin-orbit interaction between these states on the surface of crossings. This electronic structure data is crucial for any reliable treatment of this spin-forbidden reaction. We will also be considering the $\mathrm{CO}$ analogue of the $\mathrm{N}_{2}+\mathrm{O}$ system discussed above, studying from a similar perspective both $\mathrm{CO}+\mathrm{O}\left({ }^{1} \mathrm{D}\right)$ quenching and $\mathrm{CO}_{2}$ photodissociation. The later aspect of this investigation is motivated by the recent work of Stolow and Lee. ${ }^{9}$

As outlined in our original proposal we intend to consider nonadiabatic effects in excited states of the $\mathrm{HCO}$ molecule. We are currently focussing on the mechanism of predissociation in the $\widetilde{B}^{2} \mathrm{~A}^{\prime}$ system. Both spin-allowed and spin-forbidden predissociations will be considered. Our initial studies have for example located crossing of the $1^{2} \mathrm{~A}^{\prime}$ and $2^{2} \mathrm{~A}^{\prime}$ potential energy surfaces for general $\mathrm{C}_{\mathrm{S}}$ geometries. These crossings of two states of the same symmetry, which turn out not to effect predissociation of the low vibrational levels of the $\widetilde{\mathrm{B}}^{2} \mathrm{~A}^{\prime}$ state, had not been reported in previous theoretical studies of this system. Our treatment of nonadiabatic effects in this system will complement (largely DOE supported) experimental work ${ }^{10,11,12,13}$ on this system.

We presently have algorithms for directly, that is without prior determination of the potential energy surfaces in question, determining (i) the minimum energy crossing point and geometrically constrained energy minimized crossing points on the surface of intersection of two surfaces of distinct spin-multiplicity, and (ii) points on an $\mathrm{N}-2$ dimensional surface of intersection of two states of the same symmetry. Although it would appear straightforward to extend the energy minimized crossing algorithms to two states of the same symmetry problems 
associated with the abrupt switching of the identities of the states exist. In the forthcoming performance period we will attempt to develop an approach which overcomes this problem.

\section{REFERENCES}

1. D. R. Yarkony, J. Chem. Phys. 92, 2457 (1990).

2. D. R. Yarkony, J. Amer. Chem. Soc. 114, 5406 (1992).

3. D. R. Yarkony, J. Phys. Chem (1993), to appear.

4. P. Andresen, A. Bath, W. Groger, H. W. Lulf, G. Meijer, and J. J. t. Meulen, Appl. Opt. 27, 365 (1988).

5. J. A. Gray and R. L. Farrow, J. Chem. Phys. 95, 7054 (1991).

6. D. E. Heard, D. R. Crosley, J. B. Jeffries, G. P. Smith, and A. Hirano, J. Chem. Phys. 96, 4366 (1992).

7. D. R. Yarkony, J. Chem. Phys. 97, 1838 (1992).

8. S. Lee and K. F. Freed, J. Chem. Phys. 87, 5772 (1987).

9. A. Stolow and Y. T. Lee, J. Chem. Phys 98, 2066 (1993).

10. A. Sappey and D. R. Crosley, J. Chem. Phys. 93, 7601 (1990).

11. U. E. Meier, L. E. Hunziker, and D. R. Crosley, J. Phys. Chem. 95, 5163 (1991).

12. T. Cool and X.-M. Song, J. Chem. Phys 96, 8675 (1992).

13. G. W. Adamson, X. Zhao, and R. W. Field, J. Mol. Spectr. (1993), to appear. 


\section{PUBLICATIONS ACKNOWLEDGING SUPPORT OF GRANT}

\section{DE-FG02-91ER14189}

${ }^{\dagger} 1$. On the Mechanism of the Reaction $\mathrm{CH}\left(\mathrm{X}^{2} \Pi\right)+\mathrm{N}_{2}\left(\mathrm{X}^{1} \Sigma_{\mathrm{g}}^{+}\right) \rightarrow \mathrm{HCN}\left(\mathrm{X}^{1} \Sigma^{+}\right)+\mathrm{N}\left({ }^{4} S\right)$. II:The Intermediate Complex Region,

M. Riad Manaa and David R. Yarkony, Chem. Phys. Lett., 188,352 (1992).

†2 Theoretical Studies of Spin-Forbidden Radiationless Decay in Polyatomic Systems: Insights from Recently Developed Computational Methods

David R. Yarkony, J. Amer. Chem. Soc., 114, 5406, (1992)

†3. A Theroretical Treatment of the Predissociation on the Individual Rovibronic Levels of $\mathrm{OH} / \mathrm{OD}\left(\mathrm{A}^{2} \Sigma^{+}\right)$

D. R. Yarkony, J. Chem. Phys. 97, 1838 (1992).

4. Systematic Determination of Intersections of Potential Energy Surfaces Using a Lagrange Multiplier Constrained Procedure

David R. Yarkony, J. Phys. Chem. to appear

†Reprint enclosed

$\dagger$ Reprint previously provided 

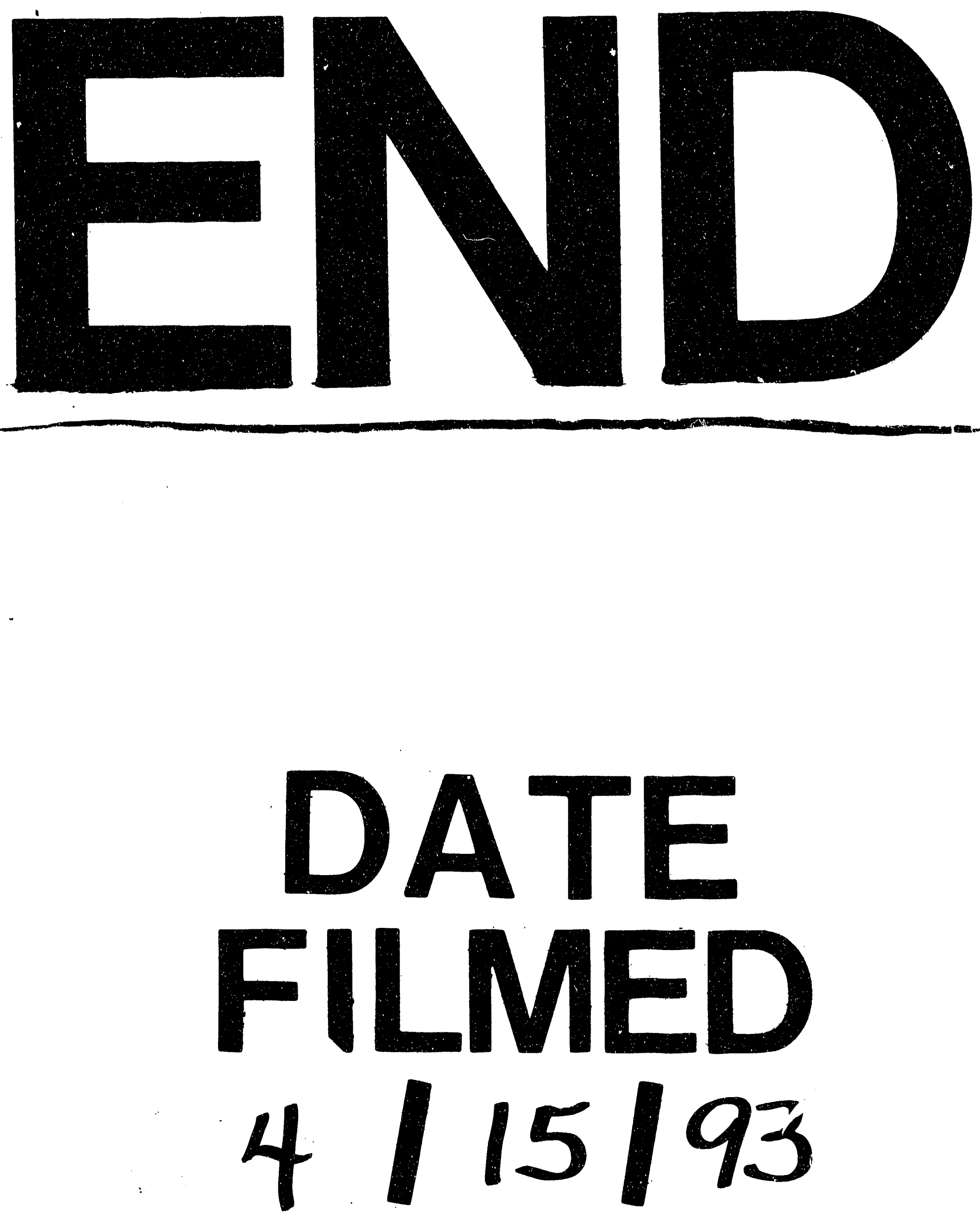
